DESY 11-063

BI-TP 2011/12

$\mathrm{SFB} / \mathrm{CPP}-11-25$

LPN 11-26

\title{
Calculating contracted tensor Feynman integrals
}

\author{
J. Fleischer $*^{* a}$ and T. Riemann $\oplus^{b}$
}

${ }^{a}$ Fakultät für Physik, Universität Bielefeld, Universitätsstr. 25, 33615 Bielefeld, Germany

${ }^{b}$ Deutsches Elektronen-Synchrotron, DESY, Platanenallee 6, 15738 Zeuthen, Germany

\begin{abstract}
A recently derived approach to the tensor reduction of 5-point one-loop Feynman integrals expresses the tensor coefficients by scalar 1-point to 4-point Feynman integrals completely algebraically. In this letter we derive extremely compact algebraic expressions for the contractions of the tensor integrals with external momenta. This is based on sums over signed minors weighted with scalar products of the external momenta. With these contractions one can construct the invariant amplitudes of the matrix elements under consideration, and the evaluation of one-loop contributions to massless and massive multi-particle production at high energy colliders like LHC and ILC is expected to be performed very efficiently.
\end{abstract}

PACS index categories: 12.15.Ji, 12.20.Ds, 12.38.Bx

\section{Introduction}

In a recent article [1] (hereafter quoted as reference I), we have worked out an algebraic method to present one-loop tensor integrals in terms of scalar one-loop 1-point to 4-point functions. The tensor integrals are defined as

$$
I_{n}^{\mu_{1} \cdots \mu_{R}}=\int \frac{d^{d} k}{i \pi^{d / 2}} \frac{\prod_{r=1}^{R} k^{\mu_{r}}}{\prod_{j=1}^{n} c_{j}},
$$

with denominators $c_{j}$, having chords $q_{j}$,

$$
c_{j}=\left(k-q_{j}\right)^{2}-m_{j}^{2}+i \varepsilon .
$$

${ }^{*}$ E-mail: fleischer@physik.uni-bielefeld.de

$\dagger$ E-mail: Tord.Riemann@desy.de 
Here, we use the generic dimension $d=4-2 \varepsilon$. The central problem are the 5-point tensor functions. We derived algebraic expressions for them in terms of higher-dimensional scalar 4-point functions with raised indices (powers of the scalar propagators). There are several ways to go. One option is to avoid the appearance of inverse Gram determinants $1 /()_{5}$. For rank $R=5$, e.g.,

$$
I_{5}^{\mu \nu \lambda \rho \sigma}=\sum_{s=1}^{5}\left[\sum_{i, j, k, l, m=1}^{5} q_{i}^{\mu} q_{j}^{v} q_{k}^{\lambda} q_{l}^{\rho} q_{m}^{\sigma} E_{i j k l m}^{s}+\sum_{i, j, k=1}^{5} g^{[\mu v} q_{i}^{\lambda} q_{j}^{\rho} q_{k}^{\sigma]} E_{00 i j k}^{s}+\sum_{i=1}^{5} g^{[\mu \nu} g^{\lambda \rho} q_{i}^{\sigma]} E_{0000 i}^{s}\right],
$$

see equations (I.4.60), (I.4.61). The tensor coefficients are expressed in terms of integrals $I_{4, i \ldots}^{[d+]^{l}, s}$, e.g. according to (I.4.62):

$$
E_{i j k l m}^{s}=-\frac{1}{\left(\begin{array}{l}
0 \\
0
\end{array}\right)_{5}}\left\{\left[\left(\begin{array}{c}
0 l \\
s m
\end{array}\right)_{5} n_{i j k} I_{4, i j k}^{[d+]^{4}, s}+(i \leftrightarrow l)+(j \leftrightarrow l)+(k \leftrightarrow l)\right]+\left(\begin{array}{c}
0 s \\
0 m
\end{array}\right)_{5} n_{i j k l} I_{4, i j k l}^{[d+]^{4}, s}\right\} .
$$

The scalar integrals are

$$
I_{p, i j k \cdots}^{[d+]^{l}, s t u \cdots}=\int \frac{d^{[d+]^{l}} k}{i \pi^{[d+]^{l} / 2}} \prod_{r=1}^{n} \frac{1}{c_{r}^{1+\delta_{r i}+\delta_{r j}+\delta_{r k}+\cdots-\delta_{r s}-\delta_{r t}-\delta_{r u}-\cdots}},
$$

where $p$ is the number of internal lines and $[d+]^{l}=4-2 \varepsilon+2 l$. Further, we use the notations of signed minors (I.2.14). At this stage, the higher-dimensional 4-point integrals still depend on tensor indices, namely through the indices $i, j$ etc. The most complicated explicit example $I_{4, i j k l}^{[d+]^{4}, s}$ appears in (1.4). Now, in a next step, one may avoid the appearance of inverse sub-Gram determinants ()$_{4}$. Indeed, after tedious manipulations, one arrives at representations in terms of scalar integrals $I_{4}^{[d+]^{l}}$ plus simpler 3-point and 2-point functions, and the complete dependence on the indices $i$ of the tensor coefficients is contained now in the pre-factors with signed minors. One can say that the indices decouple from the integrals. As an example, we reproduce the 4-point part of (I.5.21),

$$
\begin{aligned}
& n_{i j k l} I_{4, i j k l}^{[d+]^{4}}=\frac{\left(\begin{array}{l}
0 \\
i
\end{array}\right)}{\left(\begin{array}{l}
0 \\
0
\end{array}\right)} \frac{\left(\begin{array}{l}
0 \\
j
\end{array}\right)}{\left(\begin{array}{l}
0 \\
0
\end{array}\right)} \frac{\left(\begin{array}{l}
0 \\
k
\end{array}\right)}{\left(\begin{array}{l}
0 \\
0
\end{array}\right)} \frac{\left(\begin{array}{l}
0 \\
l
\end{array}\right)}{\left(\begin{array}{l}
0 \\
0
\end{array}\right)} d(d+1)(d+2)(d+3) I_{4}^{[d+]^{4}} \\
& +\frac{\left(\begin{array}{c}
0 i \\
0 j
\end{array}\right)\left(\begin{array}{l}
0 \\
k
\end{array}\right)\left(\begin{array}{l}
0 \\
l
\end{array}\right)+\left(\begin{array}{c}
0 i \\
0 k
\end{array}\right)\left(\begin{array}{l}
0 \\
j
\end{array}\right)\left(\begin{array}{l}
0 \\
l
\end{array}\right)+\left(\begin{array}{c}
0 j \\
0 k
\end{array}\right)\left(\begin{array}{l}
0 \\
i
\end{array}\right)\left(\begin{array}{l}
0 \\
l
\end{array}\right)+\left(\begin{array}{c}
0 i \\
0 l
\end{array}\right)\left(\begin{array}{l}
0 \\
j
\end{array}\right)\left(\begin{array}{l}
0 \\
k
\end{array}\right)+\left(\begin{array}{l}
0 j \\
0 l
\end{array}\right)\left(\begin{array}{l}
0 \\
i
\end{array}\right)\left(\begin{array}{l}
0 \\
k
\end{array}\right)+\left(\begin{array}{l}
0 k \\
0 l
\end{array}\right)\left(\begin{array}{l}
0 \\
i
\end{array}\right)\left(\begin{array}{l}
0 \\
j
\end{array}\right)}{\left(\begin{array}{l}
0 \\
0
\end{array}\right)^{3}} d(d+1) I_{4}^{[d+]^{3}} \\
& +\frac{\left(\begin{array}{c}
0 i \\
0 l
\end{array}\right)\left(\begin{array}{c}
0 j \\
0 k
\end{array}\right)+\left(\begin{array}{c}
0 j \\
0 l
\end{array}\right)\left(\begin{array}{c}
0 i \\
0 k
\end{array}\right)+\left(\begin{array}{c}
0 k \\
0 l
\end{array}\right)\left(\begin{array}{c}
0 i \\
0 j
\end{array}\right)}{\left(\begin{array}{l}
0 \\
0
\end{array}\right)^{2}} I_{4}^{[d+]^{2}}+\cdots
\end{aligned}
$$

In (1.6), one has to understand the 4-point integrals to carry the corresponding index $s$ and the signed minors are $\left(\begin{array}{l}0 \\ k\end{array}\right) \rightarrow\left(\begin{array}{l}0 s \\ k s\end{array}\right)_{5}$ etc. This type of relations may be called "recursion relations for small Gram determinants".

In an alternative treatment, tensor reduction formulas free of $g^{\mu v}$ terms were derived in ref. I. In that case, inverse powers of ()$_{5}$ are tolerated. The most involved object studied was (I.3.20):

$$
I_{5}^{\mu \nu \lambda \rho \sigma}=I_{5}^{\mu \nu \lambda \rho} \cdot Q_{0}^{\sigma}-\sum_{s=1}^{5} I_{4}^{\mu \nu \lambda \rho, s} \cdot Q_{s}^{\sigma},
$$


with the 4-point tensor functions (I.3.29) and (I.3.18)

$$
\begin{aligned}
I_{4}^{\mu \nu \lambda \rho, s} & =Q_{0}^{s, \mu} Q_{0}^{s, v} Q_{0}^{s, \lambda} Q_{0}^{s, \rho} I_{4}^{s}+3 \frac{()_{5}^{2}}{\left(\begin{array}{l}
s \\
s
\end{array}\right)_{5}^{2}} Q_{s}^{\mu} Q_{s}^{v} Q_{s}^{\lambda} Q_{s}^{\rho} \cdot I_{4}^{[d+]^{2}, s}+\frac{()_{5}}{\left(\begin{array}{c}
s \\
s
\end{array}\right)_{5}} Q_{s}^{[\mu} Q_{s}^{v} J_{4}^{\lambda, s} Q_{0}^{s, \rho]}+\cdots \\
J_{4}^{\mu, s} & =-Q_{0}^{s, \mu} I_{4}^{[d+]}+\sum_{t=1}^{5} Q_{t}^{s, \mu} I_{3}^{[d+], s t} .
\end{aligned}
$$

The dots in (1.8) indicate 3-point functions, and

$$
\begin{aligned}
Q_{s}^{\mu} & =\sum_{i=1}^{5} q_{i}^{\mu} \frac{\left(\begin{array}{l}
s \\
i
\end{array}\right)_{5}}{()_{5}}, \quad s=0 \cdots 5 \\
Q_{s}^{t, \mu} & =\sum_{i=1}^{5} q_{i}^{\mu} \frac{\left(\begin{array}{l}
s t \\
i t
\end{array}\right)_{5}}{\left(\begin{array}{l}
t \\
t
\end{array}\right)_{5}}, \quad s, t=1 \cdots 5 .
\end{aligned}
$$

Also here, the tensor coefficients have been represented by scalar functions free of tensor indices.

We remark that all the above-mentioned results are due to a systematic application of methods described and developed in [2]. For the present paper the "algebra of signed minors" [3] plays a particularly important role. This method was also used in [2] and further developed to its full power in [4]. In the latter article also 6-point functions have been treated on this basis.

In the next section we will develop a very efficient method to evaluate realistic matrix elements with tensor integral representations of the above kind.

\section{Contracting the tensor integrals}

To apply the approach most efficiently one should construct projection operators for the invariant amplitudes of the matrix elements under consideration. These projectors, of course, depend on the tensor basis and have to be constructed for each process specifically. If done like that, the tensor indices of the loop integrals are saturated by contractions with external momenta $p_{r}$. The chords in (1.2) are given in terms of the external momenta as $q_{i}=-\left(p_{1}+p_{2}+\cdots+p_{i}\right)$, with $q_{n}=0$, and inversely $p_{r}=q_{r-1}-q_{r}$. Then, any of the integrals to be evaluated is a simple linear combination of integrals containing products with chords, $\left(q_{r} \cdot k\right)$ :

$$
q_{i_{1} \mu_{1}} \cdots q_{i_{R} \mu_{R}} I_{5}^{\mu_{1} \cdots \mu_{R}}=\int \frac{d^{d} k}{i \pi^{d / 2}} \frac{\prod_{r=1}^{R}\left(q_{i_{r}} \cdot k\right)}{\prod_{j=1}^{5} c_{j}} .
$$

There is another type of external vectors, i.e. the polarisation vectors $\varepsilon_{i}$ of spin- 1 bosons. They, however, are taken into account in the definition of the tensor structure of the matrix elements in terms of scalar products $\left(\varepsilon_{i} \cdot p_{j}\right)$ with some external momenta $p_{j}$. The same applies to contractions with $\gamma$ matrices $\xi_{i}$ and $\not p_{i}$ in spinor chains. Thus, polarisation vectors and $\gamma$ matrices will not show up in the sums one has to perform.

If the integration momentum $k$ is self-contracted, one may use the identity $k^{2} / c_{j}=1+m_{j}^{2} / c_{j}+$ $2\left(q_{j} \cdot k\right) / c_{j}$ in order to transform the integral to the type (2.1) plus simpler ones. Since the approach uses $q_{n}=0$, one should take care that $c_{5}$ is not canceled. Then the procedure can also be applied to the scratched 4-point functions in the same manner as for the 5-point functions. Nevertheless, we do not consider this approach as optimal since in any case many new terms are produced and it seems more 
adequate also in this case to perform the corresponding sums as indicated in appendix A (see A.27A.31).

We will represent now the integrals (2.1) as compact linear combinations of scalar one-loop integrals, in higher dimensions and with indices 1 . In order to demonstrate the method, we will explicitly work out only the simplest cases with rank $R \leq 3$, but we will collect all the sums over signed minors needed also for the most complicated cases exemplified in the introduction.

The tensor 5-point integral of $\operatorname{rank} R=1$ (I.4.6) yields, when contracted with a chord,

$$
q_{a \mu} I_{5}^{\mu}=-\frac{1}{\left(\begin{array}{l}
0 \\
0
\end{array}\right)_{5}} \sum_{s=1}^{5}\left[\sum_{i=1}^{4}\left(q_{a} \cdot q_{i}\right)\left(\begin{array}{c}
0 i \\
0 s
\end{array}\right)_{5}\right] I_{4}^{s} \text {. }
$$

In fact, the sum over $i$ may be performed explicitly, it is the sum $\Sigma_{a}^{1, s}$ A.5 listed in appendix $\mathrm{A}$ and we get immediately

$$
q_{a \mu} I_{5}^{\mu}=-\frac{1}{\left(\begin{array}{l}
0 \\
0
\end{array}\right)_{5}} \sum_{s=1}^{5} \Sigma_{a}^{1, s} I_{4}^{s}
$$

The tensor 5-point integral of rank $R=2($ I.4.19),

$$
I_{5}^{\mu v}=\sum_{i, j=1}^{4} q_{i}^{\mu} q_{j}^{v} E_{i j}+g^{\mu v} E_{00}
$$

has the following tensor coefficients free of $1 /()_{5}$ :

$$
\begin{aligned}
E_{00} & =-\sum_{s=1}^{5} \frac{1}{2} \frac{1}{\left(\begin{array}{l}
0 \\
0
\end{array}\right)_{5}}\left(\begin{array}{l}
s \\
0
\end{array}\right)_{5} I_{4}^{[d+], s}, \\
E_{i j} & =\sum_{s=1}^{5} \frac{1}{\left(\begin{array}{l}
0 \\
0
\end{array}\right)_{5}}\left[\left(\begin{array}{l}
0 i \\
s j
\end{array}\right)_{5} I_{4}^{[d+], s}+\left(\begin{array}{l}
0 s \\
0 j
\end{array}\right)_{5} I_{4, i}^{[d+], s}\right] .
\end{aligned}
$$

Equation (2.4) yields for the contractions with chords:

$$
q_{a \mu} q_{b v} I_{5}^{\mu v}=\sum_{i, j=1}^{4}\left(q_{a} \cdot q_{i}\right)\left(q_{b} \cdot q_{j}\right) E_{i j}+\left(q_{a} \cdot q_{b}\right) E_{00}
$$

Applying (A.7) on the first part of (2.6), it is easy to see that the term with $\left(q_{a} \cdot q_{b}\right)$ cancels the $E_{00}$. In a next step the $I_{4, i}^{[d+], s}$ may be eliminated by a scratched version of (I.A.6) or of (I.5.10). We use here the latter one which is free of $1 /()_{4}$ :

$$
I_{4, i}^{[d+]^{l}, s}=\frac{1}{\left(\begin{array}{c}
0 s \\
0 s
\end{array}\right)_{5}}\left[-\left(\begin{array}{c}
0 s \\
i s
\end{array}\right)_{5}(d+2 l-5) I_{4}^{[d+]^{l}, s}+\sum_{t=1}^{5}\left(\begin{array}{c}
0 s t \\
0 s i
\end{array}\right)_{5} I^{[d+]^{l-1}, s t}\right], l=1,2 .
$$

We again arrived at a representation where sums over $i, j$ are decoupled from the scalar master integrals. Equations (A.5) - A.7 may be applied, and the contribution of $I_{4}^{[d+], s}$ to (2.7) reads now

$$
\frac{1}{\left(\begin{array}{l}
0 \\
0
\end{array}\right)_{5}}\left\{\left\{\Sigma_{a b}^{1, s}\right\}_{s p}-\frac{1}{\left(\begin{array}{l}
0 s \\
0 s
\end{array}\right)_{5}} \Sigma_{b}^{1, s} \cdot \Sigma_{a}^{2, s}\right\} I_{4}^{[d+], s},
$$


where $\left\{\Sigma_{a b}^{1, s}\right\}_{s p}$ is the $\left(q_{a} \cdot q_{b}\right)$-independent part of $\Sigma_{a b}^{1, s}$, A.7). The $\Sigma_{b}^{1, s}$ and $\Sigma_{a}^{2, s}$ are given in $\mathrm{A} .5$ and (A.6), respectively. A further simplification can be achieved with the identity

$$
()_{5}+\frac{\left(\begin{array}{l}
s \\
0
\end{array}\right)_{5}^{2}}{\left(\begin{array}{l}
0 s \\
0 s
\end{array}\right)_{5}}=\frac{\left(\begin{array}{l}
0 \\
0
\end{array}\right)_{5}\left(\begin{array}{l}
s \\
s
\end{array}\right)_{5}}{\left(\begin{array}{l}
0 s \\
0 s
\end{array}\right)_{5}}
$$

and finally (2.7) simply reads

$$
\begin{aligned}
q_{a \mu} q_{b v} I_{5}^{\mu \nu}= & \frac{1}{4} \sum_{s=1}^{5}\left\{\frac{\left(\begin{array}{c}
s \\
0
\end{array}\right)_{5}}{\left(\begin{array}{l}
0 s \\
0 s
\end{array}\right)_{5}}\left(\delta_{a b} \delta_{a s}+\delta_{5 s}\right)+\frac{\left(\begin{array}{c}
s \\
s
\end{array}\right)_{5}}{\left(\begin{array}{l}
0 s \\
0 s
\end{array}\right)_{5}}\left[\left(\delta_{a s}-\delta_{5 s}\right)\left(Y_{b 5}-Y_{55}\right)\right.\right. \\
& \left.\left.+\left(\delta_{b s}-\delta_{5 s}\right)\left(Y_{a 5}-Y_{55}\right)+\frac{\left(\begin{array}{c}
s \\
0
\end{array}\right)_{5}}{\left(\begin{array}{l}
0 \\
0
\end{array}\right)_{5}}\left(Y_{a 5}-Y_{55}\right)\left(Y_{b 5}-Y_{55}\right)\right]\right\} I_{4}^{[d+], s} \\
& +\frac{1}{\left(\begin{array}{l}
0 \\
0
\end{array}\right)_{5}} \sum_{s=1}^{5} \frac{\Sigma_{b}^{1, s}}{\left(\begin{array}{l}
0 s \\
0 s
\end{array}\right)_{5}} \sum_{t=1}^{5} \Sigma_{a}^{2, s t} I_{3}^{s t}
\end{aligned}
$$

with $\Sigma_{a}^{2, s t}$ given in A.11).

The result is typical in the sense that, after summation over the tensor indices, terms with factors $\left(\begin{array}{l}s \\ s\end{array}\right)_{5}$ will appear, i.e. with the Gram determinants ()$_{4}$ of the 4-point functions. This circumstance is advantageous when reducing the dimensional integrals $I_{4}^{[d+]^{l}, s}$ to lower dimensions, where factors $1 /()_{4}$ are produced. So, the problem of the small 4-point Gram determinants, discussed in great detail in ref. I, is at least partially eliminated. The remaining terms are factored by Kronecker's $\delta$-symbol and yield contributions for specific indices $a, b$ only - after summation over $s$.

Finally, we exemplify the $\operatorname{rank} R=3$ case. The tensor can be written as follows (see (I.4.35)(I.4.37)):

$$
I_{5}^{\mu \nu \lambda}=\sum_{i, j, k=1}^{5} q_{i}^{\mu} q_{j}^{v} q_{k}^{\lambda} E_{i j k}+\sum_{k=1}^{5} g^{[\mu v} q_{k}^{\lambda]} E_{00 k}
$$

with

$$
\begin{aligned}
E_{00 k} & =\sum_{s=1}^{5} \frac{1}{\left(\begin{array}{l}
0 \\
0
\end{array}\right)_{5}}\left[\frac{1}{2}\left(\begin{array}{c}
0 s \\
0 k
\end{array}\right)_{5} I_{4}^{[d+], s}-\frac{d-1}{3}\left(\begin{array}{l}
s \\
k
\end{array}\right)_{5} I_{4}^{[d+]^{2}, s}\right], \\
E_{i j k} & =-\sum_{s=1}^{5} \frac{1}{\left(\begin{array}{l}
0 \\
0
\end{array}\right)_{5}}\left\{\left[\left(\begin{array}{c}
0 j \\
s k
\end{array}\right)_{5} I_{4, i}^{[d+]^{2}, s}+(i \leftrightarrow j)\right]+\left(\begin{array}{c}
0 s \\
0 k
\end{array}\right)_{5} v_{i j} I_{4, i j}^{[d+]^{2}, s}\right\} .
\end{aligned}
$$

We eliminate now indices from scalar integrals with recursion (2.8) and further recursion relations applicable for cases with small Gram determinants ()$_{4}$, reproduced here in the unscratched forms (I.5.15) and (I.5.16):

$$
\begin{aligned}
v_{i j} I_{4, i j}^{[d+]^{2}}= & \frac{\left(\begin{array}{l}
0 \\
i
\end{array}\right)}{\left(\begin{array}{l}
0 \\
0
\end{array}\right)} \frac{\left(\begin{array}{l}
0 \\
j
\end{array}\right)}{\left(\begin{array}{l}
0 \\
0
\end{array}\right)}(d-2)(d-1) I_{4}^{[d+]^{2}}+\frac{\left(\begin{array}{c}
0 i \\
0 j
\end{array}\right)}{\left(\begin{array}{l}
0 \\
0
\end{array}\right)} I_{4}^{[d+]} \\
& -\frac{\left(\begin{array}{l}
0 \\
j
\end{array}\right)}{\left(\begin{array}{l}
0 \\
0
\end{array}\right)} \frac{d-2}{\left(\begin{array}{l}
0 \\
0
\end{array}\right)} \sum_{t=1}^{4}\left(\begin{array}{c}
0 t \\
0 i
\end{array}\right) I_{3}^{[d+], t}+\frac{1}{\left(\begin{array}{l}
0 \\
0
\end{array}\right)} \sum_{t=1}^{4}\left(\begin{array}{c}
0 t \\
0 j
\end{array}\right) I_{3, i}^{[d+], t}, \\
I_{3, i}^{[d+], t}= & -\frac{\left(\begin{array}{c}
0 t \\
i t
\end{array}\right)}{\left(\begin{array}{l}
0 t \\
0 t
\end{array}\right)}(d-2) I_{3}^{[d+], t}+\frac{1}{\left(\begin{array}{c}
0 t \\
0 t
\end{array}\right)} \sum_{u=1}^{4}\left(\begin{array}{c}
0 t u \\
0 t i
\end{array}\right) I_{2}^{t u} .
\end{aligned}
$$


The $\left(\begin{array}{l}0 t \\ 0 t\end{array}\right)$ in (2.16) vanishes for infrared divergent 3-point functions and therefore one has to use "standard" recursions a la (I.A.10) in this case. Anyway, such problems are not the concern of this letter and they have to be discussed separately if met.

After these preparations we can now evaluate the contractions of the tensor with three chords:

$$
\begin{aligned}
q_{a \mu} q_{b \nu} q_{c \lambda} I_{5}^{\mu \nu \lambda}= & \sum_{i, j, k=1}^{4}\left(q_{a} \cdot q_{i}\right)\left(q_{b} \cdot q_{j}\right)\left(q_{c} \cdot q_{k}\right) E_{i j k} \\
& +\sum_{k=1}^{4}\left[\left(q_{a} \cdot q_{b}\right)\left(q_{c} \cdot q_{k}\right)+\left(q_{a} \cdot q_{c}\right)\left(q_{b} \cdot q_{k}\right)+\left(q_{b} \cdot q_{c}\right)\left(q_{a} \cdot q_{k}\right)\right] E_{00 k} .
\end{aligned}
$$

For the triple sum over $i, j, k$ in (2.17) we get

$$
-\frac{1}{\left(\begin{array}{l}
0 \\
0
\end{array}\right)_{5}} \sum_{s=1}^{5}\left\{\Sigma_{b c}^{1, s} \cdot \sum_{i=1}^{4}\left(q_{a} \cdot q_{i}\right) I_{4, i}^{[d+]^{2}, s}+(b \leftrightarrow a)+\Sigma_{c}^{1, s} \cdot \sum_{i, j=1}^{4}\left(q_{a} \cdot q_{i}\right)\left(q_{b} \cdot q_{j}\right) v_{i j} I_{4, i j}^{[d+]^{2}, s}\right\},
$$

and get further for the sums in 2.18)

$$
\begin{aligned}
\sum_{i=1}^{4}\left(q_{a} \cdot q_{i}\right) I_{4, i}^{[d+]^{2}, s}= & \frac{1}{\left(\begin{array}{c}
0 s \\
0 s
\end{array}\right)_{5}}\left\{-\Sigma_{a}^{2, s}(d-1) I_{4}^{[d+]^{2}, s}+\sum_{t=1}^{5} \Sigma_{a}^{2, s t} I_{3}^{[d+], s t}\right\}, \\
\sum_{i, j=1}^{4}\left(q_{a} \cdot q_{i}\right)\left(q_{b} \cdot q_{j}\right) v_{i j} I_{4, i j}^{[d+]^{2}, s}= & \frac{1}{\left(\begin{array}{c}
0 s \\
0 s
\end{array}\right)_{5}^{2}} \Sigma_{a}^{2, s} \Sigma_{b}^{2, s}(d-2)(d-1) I_{4}^{[d+]^{2}, s}+\frac{1}{\left(\begin{array}{c}
0 s \\
0 s
\end{array}\right)_{5}} \Sigma_{a b}^{3, s} I_{4}^{[d+], s} \\
& -\frac{1}{\left(\begin{array}{c}
0 s \\
0 s
\end{array}\right)_{5}} \sum_{t=1}^{5}\left\{\frac{1}{\left(\begin{array}{c}
0 s \\
0 s
\end{array}\right)_{5}} \Sigma_{b}^{2, s} \Sigma_{a}^{2, s t}(d-2) I_{3}^{[d+], s t}\right. \\
& \left.+\frac{1}{\left(\begin{array}{l}
0 s t \\
0 s t
\end{array}\right)_{5}} \Sigma_{b}^{2, s t}\left[\Sigma_{a}^{3, s t}(d-2) I_{3}^{[d+], s t}-\sum_{u=1}^{5} \Sigma_{a}^{2, s t u} I_{2}^{s t u}\right]\right\} .
\end{aligned}
$$

Finally, for the single sum in 2.17) we have

$$
\sum_{k=1}^{4}\left(q_{c} \cdot q_{k}\right) E_{00 k}^{s}=\frac{1}{2\left(\begin{array}{l}
0 \\
0
\end{array}\right)_{5}}\left[\Sigma_{c}^{1, s} I_{4}^{[d+], s}-\frac{d-1}{3}()_{5}\left(\delta_{c s}-\delta_{5 s}\right) I_{4}^{[d+]^{2}, s}\right] .
$$

We leave the task to collect the terms needed in (2.17) to the reader. We only mention that similar simplifications like those for the tensor of rank $R=2$ can be achieved if one evaluates (2.17) with a symmetrized version of (2.14).

It is interesting to compare our approach with the so called OPP method [5, 6]. For this purpose we concentrate on the 5-point function, which was discussed in detail so far. Both methods start from a recursion relation, namely (2.2) in [5] and (I.2.5), derived in [7]. In further steps, of course, we do not identify the results, but find analogies.

The first analogy is the representation of the 5-point tensor by means of the number of scalar propagators, resulting in 4-, 3-, 2- and 1-point functions. This is given in [6] by (1.1) and (1.2). In the present work we use representations where the tensors are reduced correspondingly to 4-, 3-, 2- and 1-point integrals (with indices 1), however the integrations are performed in general in higher spacetime dimensions. In fact there will occur, in general, even several integrals in different dimension, like e.g. in (1.6). One essential difference is that in our approach there is no 0-point, "spurious" contribution: performing recursions, these terminate with 1-point functions; see e.g. appendix A of ref. I. 
The next step is the analogy of the coefficients $d, c, b, a$ in [6] and ours, given in ref. I. Taking again (1.6) as an example, our coefficients are written explicitly in terms of signed minors - as can be seen from ref. I for the 3-, 2- and 1-point functions as well. This means we do not need a recursion going down the chain $d, c, b, a$. Instead, we have solved the recursion. In [6] the tensor indices are carried by massless 4 -vectors $l_{1} \ldots l_{4}$ while in our case they are carried by the chords $q_{i}$.

In [6] the coefficients $d, c, b, a$ are calculated numerically, while here they are given analytically. So we can go one step further and perform the summation over the indices as demonstrated above and in detail in appendix A. In fact, relying on projectors to obtain the invariant amplitudes of a matrix element, these sums are at most two-fold. The reason that there are no further sums to be evaluated is due to the fact that not only the indices decouple from the integrals, but in addtion to that they also factorize such that at most two indices occur in any one signed minor.

\section{Conclusions}

The contracted rank $R=1 \cdots 3$ tensors of the 5-point function have been expressed by scalar integrals, accompanied by compact expressions for sums over products of chords and signed minors. It is evident how the general case has to be treated, once a table of sums as given in the appendix is available. The scalar integrals may be defined in higher dimensions or in the generic dimension, depending on the preferred algorithm of the final numerical evaluations and on questions related to a treatment (or avoidance) of inverse Gram determinants.

Based on the approach defined in this letter, we expect a considerable economization of crosssection calculations in cases where an essential part of the computational time and storage is spent on tensor reduction.

\section{Acknowledgements}

We acknowledge useful discussions with S. Moch and P. Uwer. J.F. thanks DESY for kind hospitality. Work is supported in part by Sonderforschungsbereich/Transregio SFB/TRR 9 of DFG "Computergestützte Theoretische Teilchenphysik" and European Initial Training Network LHCPHENOnet PITNGA-2010-264564.

\section{A Sums over contracted chords and signed minors}

A useful notation is

$$
Y_{i j}=-\left(q_{i}-q_{j}\right)^{2}+m_{i}^{2}+m_{j}^{2} .
$$

The simplest contractions are given in [7]:

$$
\begin{aligned}
\left(q_{i} \cdot Q_{0}\right) & =\sum_{j=1}^{n-1}\left(q_{i} \cdot q_{j}\right) \frac{\left(\begin{array}{l}
0 \\
j
\end{array}\right)_{n}}{()_{n}}=-\frac{1}{2}\left(Y_{i n}-Y_{n n}\right), \quad i=1, \ldots, n-1, \\
\left(q_{i} \cdot Q_{s}\right) & =\sum_{j=1}^{n-1}\left(q_{i} \cdot q_{j}\right) \frac{\left(\begin{array}{l}
s \\
j
\end{array}\right)_{n}}{()_{n}}=\frac{1}{2}\left(\delta_{i s}-\delta_{n s}\right), \quad i=1, \ldots, n-1, \quad s=1, \ldots n .
\end{aligned}
$$


The $Q_{s}, Q_{0}$ are defined in (1.10). In (A.2) and (A.3), $q_{n}=0$ is assumed since only in this case the relation

$$
\left(q_{i} \cdot q_{j}\right)=\frac{1}{2}\left[Y_{i j}-Y_{i n}-Y_{n j}+Y_{n n}\right]
$$

holds which is needed for their derivations.

Further sums are needed if the 4-point tensors are contracted:

$$
\begin{aligned}
& \Sigma_{a}^{1, s} \equiv \sum_{i=1}^{4}\left(q_{a} \cdot q_{i}\right)\left(\begin{array}{c}
0 s \\
0 i
\end{array}\right)_{5}=+\frac{1}{2}\left\{\left(\begin{array}{l}
s \\
0
\end{array}\right)_{5}\left(Y_{a 5}-Y_{55}\right)+\left(\begin{array}{l}
0 \\
0
\end{array}\right)_{5}\left(\delta_{a s}-\delta_{5 s}\right)\right\} \\
& \Sigma_{a}^{2, s} \equiv \sum_{i=1}^{4}\left(q_{a} \cdot q_{i}\right)\left(\begin{array}{l}
0 s \\
i s
\end{array}\right)_{5}=-\frac{1}{2}\left\{\left(\begin{array}{l}
s \\
s
\end{array}\right)_{5}\left(Y_{a 5}-Y_{55}\right)+\left(\begin{array}{l}
s \\
0
\end{array}\right)_{5}\left(\delta_{a s}-\delta_{5 s}\right)\right\} .
\end{aligned}
$$

Double sums for 4-point functions:

$$
\begin{aligned}
\Sigma_{a b}^{1, s} \equiv & \sum_{i, j=1}^{4}\left(q_{a} \cdot q_{i}\right)\left(q_{b} \cdot q_{j}\right)\left(\begin{array}{c}
0 i \\
s j
\end{array}\right)_{5}=\frac{1}{2}\left(q_{a} \cdot q_{b}\right)\left(\begin{array}{l}
s \\
0
\end{array}\right)_{5}+\frac{1}{4}()_{5}\left(Y_{b 5}-Y_{55}\right)\left(\delta_{a s}-\delta_{5 s}\right) \\
\Sigma_{a b}^{2, s} \equiv & \sum_{i, j=1}^{4}\left(q_{a} \cdot q_{i}\right)\left(q_{b} \cdot q_{j}\right)\left(\begin{array}{c}
s i \\
s j
\end{array}\right)_{5}=\frac{1}{2}\left(q_{a} \cdot q_{b}\right)\left(\begin{array}{l}
s \\
s
\end{array}\right)_{5}-\frac{1}{4}()_{5}\left(\delta_{a b} \delta_{a s}+\delta_{5 s}\right) \\
\Sigma_{a b}^{3, s} \equiv & \sum_{i, j=1}^{4}\left(q_{a} \cdot q_{i}\right)\left(q_{b} \cdot q_{j}\right)\left(\begin{array}{c}
0 s i \\
0 s j
\end{array}\right)_{5}=\frac{1}{2}\left(q_{a} \cdot q_{b}\right)\left(\begin{array}{c}
0 s \\
0 s
\end{array}\right)_{5}-\frac{1}{4}\left\{\left(\begin{array}{l}
s \\
s
\end{array}\right)_{5}\left(Y_{a 5}-Y_{55}\right)\left(Y_{b 5}-Y_{55}\right)\right. \\
& \left.+\left(\begin{array}{l}
s \\
0
\end{array}\right)_{5}\left[\left(\delta_{a s}-\delta_{5 s}\right)\left(Y_{b 5}-Y_{55}\right)+\left(\delta_{b s}-\delta_{5 s}\right)\left(Y_{a 5}-Y_{55}\right)\right]+\left(\begin{array}{l}
0 \\
0
\end{array}\right)_{5}\left(\delta_{a b} \delta_{a s}+\delta_{5 s}\right)\right\}
\end{aligned}
$$

Sums for 3-point functions:

$$
\begin{aligned}
\Sigma_{a}^{1, s t} & \equiv \sum_{i=1}^{4}\left(q_{a} \cdot q_{i}\right)\left(\begin{array}{c}
t s \\
i s
\end{array}\right)_{5}=\frac{1}{2}\left(1-\delta_{s t}\right)\left\{\left(\begin{array}{l}
s \\
s
\end{array}\right)_{5}\left(\delta_{a t}-\delta_{5 t}\right)-\left(\begin{array}{c}
s \\
t
\end{array}\right)_{5}\left(\delta_{a s}-\delta_{5 s}\right)\right\}, \\
\Sigma_{a}^{2, s t} & \equiv \sum_{i=1}^{4}\left(q_{a} \cdot q_{i}\right)\left(\begin{array}{c}
0 s t \\
0 s i
\end{array}\right)_{5} \\
& =\frac{1}{2}\left(1-\delta_{s t}\right)\left\{\left(\begin{array}{c}
t s \\
0 s
\end{array}\right)_{5}\left(Y_{a 5}-Y_{55}\right)+\left(\begin{array}{c}
0 s \\
0 s
\end{array}\right)_{5}\left(\delta_{a t}-\delta_{5 t}\right)-\left(\begin{array}{c}
0 s \\
0 t
\end{array}\right)_{5}\left(\delta_{a s}-\delta_{5 s}\right)\right\}, \\
\Sigma_{a}^{3, s t} & \equiv \sum_{i=1}^{4}\left(q_{a} \cdot q_{i}\right)\left(\begin{array}{c}
0 s t \\
i s t
\end{array}\right)_{5} \\
& =-\frac{1}{2}\left\{\left(\begin{array}{c}
s t \\
s t
\end{array}\right)_{5}\left(Y_{a 5}-Y_{55}\right)+\left(\begin{array}{c}
s t \\
s 0
\end{array}\right)_{5}\left(\delta_{a t}-\delta_{5 t}\right)+\left(\begin{array}{c}
s t \\
0 t
\end{array}\right)_{5}\left(\delta_{a s}-\delta_{5 s}\right)\right\} .
\end{aligned}
$$


Double sums for 3-point functions:

$$
\begin{aligned}
& \Sigma_{a b}^{4, s t} \equiv \sum_{i, j=1}^{4}\left(q_{a} \cdot q_{i}\right)\left(q_{b} \cdot q_{j}\right)\left(\begin{array}{c}
0 s t i \\
0 s t j
\end{array}\right)_{5}=\frac{1}{2}\left(q_{a} \cdot q_{b}\right)\left(\begin{array}{c}
0 s t \\
0 s t
\end{array}\right)_{5}-\frac{1}{4}\left(1-\delta_{s t}\right) \\
& \times\left\{\left(\begin{array}{c}
s t \\
s t
\end{array}\right)_{5}\left(Y_{a 5}-Y_{55}\right)\left(Y_{b 5}-Y_{55}\right)+\left(\begin{array}{c}
0 s \\
0 s
\end{array}\right)_{5}\left(\delta_{a b} \delta_{a t}+\delta_{5 t}\right)+\left(\begin{array}{c}
0 t \\
0 t
\end{array}\right)_{5}\left(\delta_{a b} \delta_{a s}+\delta_{5 s}\right)\right. \\
& +\left(\begin{array}{l}
s t \\
s 0
\end{array}\right)_{5}\left[\left(\delta_{a t}-\delta_{5 t}\right)\left(Y_{b 5}-Y_{55}\right)+\left(\delta_{b t}-\delta_{5 t}\right)\left(Y_{a 5}-Y_{55}\right)\right] \\
& +\left(\begin{array}{c}
s t \\
0 t
\end{array}\right)_{5}\left[\left(\delta_{a s}-\delta_{5 s}\right)\left(Y_{b 5}-Y_{55}\right)+\left(\delta_{b s}-\delta_{5 s}\right)\left(Y_{a 5}-Y_{55}\right)\right] \\
& \left.-\left(\begin{array}{c}
0 s \\
0 t
\end{array}\right)_{5}\left[\left(\delta_{a t}-\delta_{5 t}\right)\left(\delta_{b s}-\delta_{5 s}\right)+\left(\delta_{b t}-\delta_{5 t}\right)\left(\delta_{a s}-\delta_{5 s}\right)\right]\right\}, \\
& \Sigma_{a b}^{4, s t u} \equiv \sum_{i, j=1}^{4}\left(q_{a} \cdot q_{i}\right)\left(q_{b} \cdot q_{j}\right)\left(\begin{array}{c}
s t u i \\
s t u j
\end{array}\right)_{5}=\frac{1}{2}\left(q_{a} \cdot q_{b}\right)\left(\begin{array}{l}
s t u \\
s t u
\end{array}\right)_{5}-\frac{1}{4}\left(1-\delta_{s t}\right)\left(1-\delta_{s u}\right)\left(1-\delta_{t u}\right) \\
& \left\{\left(\begin{array}{l}
s t \\
s t
\end{array}\right)_{5}\left(\delta_{a b} \delta_{a u}+\delta_{5 u}\right)+\left(\begin{array}{l}
s u \\
s u
\end{array}\right)_{5}\left(\delta_{a b} \delta_{a t}+\delta_{5 t}\right)+\left(\begin{array}{l}
t u \\
t u
\end{array}\right)_{5}\left(\delta_{a b} \delta_{a s}+\delta_{5 s}\right)\right. \\
& -\left(\begin{array}{l}
s t \\
s u
\end{array}\right)_{5}\left[\left(\delta_{a t}-\delta_{5 t}\right)\left(\delta_{b u}-\delta_{5 u}\right)+\left(\delta_{b t}-\delta_{5 t}\right)\left(\delta_{a u}-\delta_{5 u}\right)\right] \\
& -\left(\begin{array}{c}
t s \\
t u
\end{array}\right)_{5}\left[\left(\delta_{a u}-\delta_{5 u}\right)\left(\delta_{b s}-\delta_{5 s}\right)+\left(\delta_{b u}-\delta_{5 u}\right)\left(\delta_{a s}-\delta_{5 s}\right)\right] \\
& \left.-\left(\begin{array}{l}
u s \\
u t
\end{array}\right)_{5}\left[\left(\delta_{a t}-\delta_{5 t}\right)\left(\delta_{b s}-\delta_{5 s}\right)+\left(\delta_{b t}-\delta_{5 t}\right)\left(\delta_{a s}-\delta_{5 s}\right)\right]\right\} .
\end{aligned}
$$

Sums for 2-point functions:

$$
\begin{aligned}
\Sigma_{a}^{1, s t u} \equiv & \sum_{i=1}^{4}\left(q_{a} \cdot q_{i}\right)\left(\begin{array}{c}
s t u \\
s t i
\end{array}\right)_{5}=\frac{1}{2}\left(1-\delta_{s u}\right)\left(1-\delta_{t u}\right) \\
& \times\left\{\left(\begin{array}{c}
s t \\
s t
\end{array}\right)_{5}\left(\delta_{a u}-\delta_{5 u}\right)-\left(\begin{array}{c}
s t \\
s u
\end{array}\right)_{5}\left(\delta_{a t}-\delta_{5 t}\right)-\left(\begin{array}{c}
t s \\
t u
\end{array}\right)_{5}\left(\delta_{a s}-\delta_{5 s}\right)\right\} \\
\Sigma_{a}^{2, s t u} \equiv & \sum_{i=1}^{4}\left(q_{a} \cdot q_{i}\right)\left(\begin{array}{c}
0 s t u \\
0 s t i
\end{array}\right)_{5}=\frac{1}{2}\left(1-\delta_{s u}\right)\left(1-\delta_{t u}\right)\left\{\left(\begin{array}{c}
s t u \\
s t 0
\end{array}\right)_{5}\left(Y_{a 5}-Y_{55}\right)\right. \\
& \left.+\left(\begin{array}{c}
0 s t \\
0 s t
\end{array}\right)_{5}\left(\delta_{a u}-\delta_{5 u}\right)-\left(\begin{array}{c}
0 s t \\
0 s u
\end{array}\right)_{5}\left(\delta_{a t}-\delta_{5 t}\right)-\left(\begin{array}{c}
0 t s \\
0 t u
\end{array}\right)_{5}\left(\delta_{a s}-\delta_{5 s}\right)\right\} \\
\Sigma_{a}^{3, s t u} \equiv & \sum_{i=1}^{4}\left(q_{a} \cdot q_{i}\right)\left(\begin{array}{c}
0 s t u \\
i s t u
\end{array}\right)_{5}=-\frac{1}{2}\left\{\begin{array}{l}
s t u \\
s t u
\end{array}\right)_{5}\left(Y_{a 5}-Y_{55}\right) \\
& \left.+\left(\begin{array}{c}
s t u \\
0 t u
\end{array}\right)_{5}\left(\delta_{a s}-\delta_{5 s}\right)+\left(\begin{array}{c}
t s u \\
0 s u
\end{array}\right)_{5}\left(\delta_{a t}-\delta_{5 t}\right)+\left(\begin{array}{c}
u s t \\
0 s t
\end{array}\right)_{5}\left(\delta_{a u}-\delta_{5 u}\right)\right\}
\end{aligned}
$$


Sums for 1-point functions:

$$
\begin{aligned}
\Sigma_{a}^{1, s t u v} \equiv & \sum_{i=1}^{4}\left(q_{a} \cdot q_{i}\right)\left(\begin{array}{c}
v s t u \\
i s t u
\end{array}\right)_{5}=\frac{1}{2}\left(1-\delta_{s v}\right)\left(1-\delta_{t v}\right)\left(1-\delta_{u v}\right)\left\{\left(\begin{array}{l}
s t u \\
s t u
\end{array}\right)_{5}\left(\delta_{a v}-\delta_{5 v}\right)\right. \\
& \left.-\left(\begin{array}{c}
s t u \\
s t v
\end{array}\right)_{5}\left(\delta_{a u}-\delta_{5 u}\right)-\left(\begin{array}{c}
s u t \\
s u v
\end{array}\right)_{5}\left(\delta_{a t}-\delta_{5 t}\right)-\left(\begin{array}{c}
t u s \\
t u v
\end{array}\right)_{5}\left(\delta_{a s}-\delta_{5 s}\right)\right\}, \\
\Sigma_{a}^{2, s t u v} \equiv & \sum_{i=1}^{4}\left(q_{a} \cdot q_{i}\right)\left(\begin{array}{c}
0 s t u v \\
i s t u v
\end{array}\right)_{5}=-\frac{1}{2}\left\{\left(\begin{array}{c}
s t u v \\
s t u v
\end{array}\right)_{5}\left(Y_{a 5}-Y_{55}\right)+\left(\begin{array}{c}
s t u v \\
0 t u v
\end{array}\right)_{5}\left(\delta_{a s}-\delta_{5 s}\right)\right. \\
& \left.+\left(\begin{array}{c}
t s u v \\
0 s u v
\end{array}\right)_{5}\left(\delta_{a t}-\delta_{5 t}\right)+\left(\begin{array}{c}
u s t v \\
0 s t v
\end{array}\right)_{5}\left(\delta_{a u}-\delta_{5 u}\right)+\left(\begin{array}{c}
v s t u \\
0 s t u
\end{array}\right)_{5}\left(\delta_{a v}-\delta_{5 v}\right)\right\} .
\end{aligned}
$$

The sums A.10 - A.19 vanish whenever two of the indices $s, t, u, v$ are equal. Nevertheless, in order to underline this property, we have occasionally introduced factors $\left(1-\delta_{s t}\right) \cdots$ in front of the curly brackets when the vanishing of the right hand side of these equations for equal indices is not so obvious and comes about due to a cancellation. Keeping this in mind we can give some simpler representations for A.17 - A.19 due to the many scratches in the signed minors. With $w=10-s-t-u$ and $x=15-s-t-u-v=w+(5-v)$, a detailed investigation shows that

$$
\begin{array}{rlrl}
\Sigma_{a}^{3, \text { stu }} & =\left(Y_{w 5}-Y_{55}\right)\left(q_{a} \cdot q_{w}\right), & & s, t, u=1, \ldots, 4, \\
\Sigma_{a}^{1, s t u v} & =-\left(q_{a} \cdot q_{v}\right), & s, t, u, v=1, \ldots, 4, \\
& =\left(q_{a} \cdot q_{x}\right), & v & =5, \\
& =-\left(q_{a} \cdot q_{v}\right)+\left(q_{a} \cdot q_{x}\right), & s, t, u=5, \\
\Sigma_{a}^{2, s t u v} & =0, & s, t, u, v=1, \ldots, 4, \\
& =-\left(q_{a} \cdot q_{x}\right), & s, t, u, v=5 .
\end{array}
$$

Coefficients A.20) multiply $I_{2}\left(m_{w}, m_{5}\right)(w=1, \ldots, 4)$ and A.21, A.22 multiply $I_{1}\left(m_{5}\right)(s, t, u, v=$ $1, \ldots, 4)$ and $I_{1}\left(m_{x}\right)(x=1, \ldots, 4)$ if one of the indices $s, t, u, v$ is equal to 5 . In the other cases one better keeps the notation in terms of signed minors.

The above sums are complete in the sense that no more sums occur if the integrals are contracted with external momenta. Other sums, however, can occur e.g. if the integration momentum is selfcontracted or if, in special investigations, in the above double sums one of the indices remains uncontracted. Since not all these sums can be dealt with in this letter, we scetch their formal derivation.

In principle, the only relation needed is found in [3],

$$
()_{n}\left(\begin{array}{c}
i k \\
j l
\end{array}\right)_{n}=\left(\begin{array}{l}
i \\
j
\end{array}\right)_{n}\left(\begin{array}{l}
k \\
l
\end{array}\right)_{n}-\left(\begin{array}{l}
i \\
l
\end{array}\right)_{n}\left(\begin{array}{l}
j \\
k
\end{array}\right)_{n} .
$$

Let us prove (A.10) as an example. We write

$$
\begin{aligned}
()_{5} \sum_{i=1}^{4}\left(q_{a} \cdot q_{i}\right)\left(\begin{array}{l}
t s \\
i s
\end{array}\right)_{5} & =\sum_{i=1}^{4}\left(q_{a} \cdot q_{i}\right)\left[\left(\begin{array}{l}
t \\
i
\end{array}\right)_{5}\left(\begin{array}{l}
s \\
s
\end{array}\right)_{5}-\left(\begin{array}{l}
s \\
i
\end{array}\right)_{5}\left(\begin{array}{l}
s \\
t
\end{array}\right)_{5}\right] \\
& =\left(\begin{array}{l}
s \\
s
\end{array}\right)_{5} \sum_{i=1}^{4}\left(q_{a} \cdot q_{i}\right)\left(\begin{array}{l}
t \\
i
\end{array}\right)_{5}-\left(\begin{array}{l}
s \\
t
\end{array}\right)_{5} \sum_{i=1}^{4}\left(q_{a} \cdot q_{i}\right)\left(\begin{array}{l}
s \\
i
\end{array}\right)_{5}
\end{aligned}
$$


With (A.2) and (A.3) we see that ()$_{5}$ cancels and (A.10) is obtained. The factor $\left(1-\delta_{s t}\right)$ only stresses the fact that for $s=t$ the signed minor $\left(\begin{array}{c}t s \\ i s\end{array}\right)_{5}$ vanishes and so does the sum.

The same procedure also applies for the other sums. Let us look at (A.12). We have to take into account that (A.23) applies for any $n$, i.e. it is also valid if any row and column with the same index, say $s$, is scratched. This would give

$$
\left(\begin{array}{l}
s \\
s
\end{array}\right)_{5}\left(\begin{array}{l}
0 s t \\
i s t
\end{array}\right)_{5}=\left(\begin{array}{c}
0 s \\
i s
\end{array}\right)_{5}\left(\begin{array}{l}
s t \\
s t
\end{array}\right)_{5}-\left(\begin{array}{c}
t s \\
0 s
\end{array}\right)_{5}\left(\begin{array}{c}
t s \\
i s
\end{array}\right)_{5}
$$

Such relations are called extensionals in [3]. We now write correspondingly

$$
\begin{aligned}
\left(\begin{array}{c}
s \\
s
\end{array}\right)_{5} \sum_{i=1}^{4}\left(q_{a} \cdot q_{i}\right)\left(\begin{array}{c}
0 s t \\
i s t
\end{array}\right)_{5} & =\sum_{i=1}^{4}\left(q_{a} \cdot q_{i}\right)\left[\left(\begin{array}{c}
0 s \\
i s
\end{array}\right)_{5}\left(\begin{array}{c}
s t \\
s t
\end{array}\right)_{5}-\left(\begin{array}{c}
t s \\
0 s
\end{array}\right)_{5}\left(\begin{array}{c}
t s \\
i s
\end{array}\right)_{5}\right] \\
& =\left(\begin{array}{c}
s t \\
s t
\end{array}\right)_{5} \sum_{i=1}^{4}\left(q_{a} \cdot q_{i}\right)\left(\begin{array}{c}
0 s \\
i s
\end{array}\right)_{5}-\left(\begin{array}{c}
t s \\
0 s
\end{array}\right)_{5} \sum_{i=1}^{4}\left(q_{a} \cdot q_{i}\right)\left(\begin{array}{c}
t s \\
i s
\end{array}\right)_{5} .
\end{aligned}
$$

The sums appearing here are A.6 and A.10). Inserting these sums, some algebra shows that the factor $\left(\begin{array}{l}s \\ s\end{array}\right)_{5}$ can be canceled and (A.12) is obtained.

The approach is quite general: we multiply the sums under consideration with the proper Gram determinant such that an extensional of A.23 can be applied. This reduces the entries in the signed minors to be summed over such that the obtained sums have signed minors with less entries and are known from former steps. The Gram determinant multiplying the original sum must cancel at the end after some algebra. In this way any sum can be obtained by iteration.

We can now scetch how self-contracted integration momenta can be dealt with. Some "start-up“ sums are (I.7.16)-(I.7.17), (I.7.20)-(I.7.22). These sums present the type of self-contracted integration momenta. The (7.16) and (7.17), e.g., read

$$
\begin{aligned}
\sum_{i, j=1}^{4}\left(q_{i} \cdot q_{j}\right)\left(\begin{array}{c}
0 s \\
i s
\end{array}\right)_{5}\left(\begin{array}{c}
0 s \\
j s
\end{array}\right)_{5} & =\frac{1}{2}\left(\begin{array}{l}
s \\
s
\end{array}\right)_{5}\left[\left(\begin{array}{c}
0 s \\
0 s
\end{array}\right)_{5}+Y_{55}\left(\begin{array}{l}
s \\
s
\end{array}\right)_{5}+2\left(\begin{array}{l}
s \\
0
\end{array}\right)_{5} \delta_{5 s}\right], \\
\sum_{i, j=1}^{4}\left(q_{i} \cdot q_{j}\right)\left(\begin{array}{c}
i s \\
j s
\end{array}\right)_{5} & =\frac{3}{2}\left(\begin{array}{l}
s \\
s
\end{array}\right)_{5}
\end{aligned}
$$

In fact, due to (1.6) the sum (A.27) is already one of the sums occurring if the vectors $q_{i}$ and $q_{j}$ are contracted. A further sum might be

$$
\sum_{i, j=1}^{4}\left(q_{i} \cdot q_{j}\right)\left(\begin{array}{c}
0 s i \\
0 s j
\end{array}\right)_{5}
$$

With

$$
\left(\begin{array}{l}
s \\
s
\end{array}\right)_{5}\left(\begin{array}{c}
0 s i \\
0 s j
\end{array}\right)_{5}=\left(\begin{array}{c}
0 s \\
0 s
\end{array}\right)_{5}\left(\begin{array}{c}
i s \\
j s
\end{array}\right)_{5}-\left(\begin{array}{c}
0 s \\
i s
\end{array}\right)_{5}\left(\begin{array}{c}
0 s \\
j s
\end{array}\right)_{5}
$$

we see that (A.29) can be reduced to (A.27) and with the final result

$$
\sum_{i, j=1}^{4}\left(q_{i} \cdot q_{j}\right)\left(\begin{array}{c}
0 s i \\
0 s j
\end{array}\right)_{5}=\left(\begin{array}{c}
0 s \\
0 s
\end{array}\right)_{5}-\frac{1}{2}\left(\begin{array}{l}
s \\
s
\end{array}\right)_{5} Y_{55}-\left(\begin{array}{l}
s \\
0
\end{array}\right)_{5} \delta_{5 s} .
$$

In this manner the self-contracted integration momenta can be dealt with like the other ones, coming from contractions with external momenta, and thus provide a consistent picture of our approach. 


\section{References}

[1] J. Fleischer, T. Riemann, Complete algebraic reduction of one-loop tensor Feynman integrals, Phys. Rev. D83 (2011) 073004. arXiv:1009.4436, doi:10.1103/PhysRevD.83.073004.

[2] J. Fleischer, F. Jegerlehner, O. Tarasov, Algebraic reduction of one-loop Feynman graph amplitudes, Nucl. Phys. B566 (2000) 423-440. arXiv:hep-ph/9907327. doi:10.1016/S0550-3213(99)00678-1.

[3] D. B. Melrose, Reduction of Feynman diagrams, Nuovo Cim. 40 (1965) 181-213. doi:10.1007/BF028329.

[4] T. Diakonidis, J. Fleischer, J. Gluza, K. Kajda, T. Riemann, J. Tausk, A complete reduction of one-loop tensor 5- and 6-point integrals, Phys. Rev. D80 (2009) 036003. arXiv:0812.2134, doi:10.1103/PhysRevD.80.036003

[5] F. del Aguila, R. Pittau, Recursive numerical calculus of one-loop tensor integrals, JHEP 0407 (2004) 017, erratum added online, feb/4/2005. arXiv: hep-ph/0404120, doi:10.1088/1126-6708/2004/07/017.

[6] G. Ossola, C. Papadopoulos, R. Pittau, Reducing full one-loop amplitudes to scalar integrals at the integrand level, Nucl. Phys. B763 (2007) 147-169. arXiv:hep-ph/0609007. doi:10.1016/j.nuclphysb.2006.11.012.

[7] T. Diakonidis, J. Fleischer, T. Riemann, J. B. Tausk, A recursive reduction of tensor Feynman integrals, Phys. Lett. B683 (2010) 69-74. arXiv:0907.2115, doi:10.1016/j.physletb.2009.11.049. 